\title{
High-temperature Mott transition in wide-band-gap semiconductor quantum wells
}

\author{
G. Rossbach, ' J. Levrat, G. Jacopin, M. Shahmohammadi, J.-F. Carlin, J.-D. Ganière, R. Butté, B. Deveaud, and N. Grandjean \\ Institute of Condensed Matter Physics, École Polytechnique Fédérale de Lausanne (EPFL), CH-1015 Lausanne, Switzerland
}

(Received 20 December 2013; revised manuscript received 22 October 2014; published 19 November 2014)

\begin{abstract}
The crossover from an exciton gas to an electron-hole plasma is studied in a GaN/(Al,Ga)N single quantum well by means of combined time-resolved and continuous-wave photoluminescence measurements. The twodimensional Mott transition is found to be of continuous type and to be accompanied by a characteristic modification of the quantum well emission spectrum. Beyond the critical density, the latter is strongly influenced by band-gap renormalization and Fermi filling of continuum states. Owing to the large binding energy of excitons in III-nitride heterostructures, their injection-induced dissociation could be tracked over a wide range of temperatures, i.e., from 4 to $150 \mathrm{~K}$. Various criteria defining the Mott transition are examined, which, however, do not lead to any clear trend with rising temperature: the critical carrier density remains invariant around $10^{12} \mathrm{~cm}^{-2}$.
\end{abstract}

DOI: 10.1103/PhysRevB.90.201308

PACS number(s): 78.67.De, 71.10.Hf, 71.35.Cc

At sufficiently low temperature $T$ and carrier density $n$, free electrons and holes in a semiconductor bind and form neutrally charged quasiparticles. These so-called excitons represent the fundamental electronic excitation of a semiconductor and obey Bose statistics in the low-density limit. However, when increasing $T$ or $n$ beyond a certain limit, the exciton complexes get ionized and the system switches from an insulating state to a conductive electron-hole plasma (EHP)—the Mott transition (MT) [1]. While exciton dissociation induced by a hot phonon bath represents a classical process and occurs when the thermal energy becomes of the order of the exciton binding energy $E_{\mathrm{X}}^{\mathrm{b}} \approx k_{\mathrm{B}} T$, the breakup of excitons due to an increasing carrier population relies on much more complex mechanisms. In simplified terms, when $n$ approaches the hard-sphere limit, that is, when the interparticle distance is reduced to the order of the exciton Bohr radius $a_{\mathrm{B}}$, the fermionic properties of the exciton constituents become dominant: Coulomb screening and phase-space filling cause a reduction of $E_{\mathrm{X}}^{\mathrm{b}}$ and eventually the dissociation of the excitonic bound state around a certain critical density $n_{\text {crit }}$ [2]. Note that the latter is usually overestimated by the simple hard-sphere criterion [3].

Whereas the MT was initially claimed to be a first-order phase transition $[1,4]$, later experiments led to partially conflicting results. Especially in three dimensions (3D), studies exist that argue to evidence the first-order nature of the MT [5,6], while others point toward a second-order transition [7-9]. However, note that in optically-probed bulk systems, the exponential absorption profile leads to an emission signal that mixes inhomogeneously injected regions and may conceal certain characteristics of the MT. This drawback can be circumvented in 2D systems. Here, most of the studies suggest a rather smooth MT [10-13]. On the contrary, experimental studies concerning the $T$ dependence of the MT are extremely scarce. In a simple framework, an increasing Debye-screening length gives reason to expect a rise in $n_{\text {crit }}$ with $T$. Even if such a behavior was claimed for bulk Ge in Ref. [8], the situation remains partly controversial [14], e.g., due to the inconsistencies arising when the MT is described by static screening [3], and the expected impact of dynamic screening effects $[2,15]$.

*georg.rossbach@epfl.ch
Hence, the MT and in particular its $T$ dependence require further experimental verification: a deeper insight is important for a variety of fields. For instance, cavity-polariton condensation takes place below the MT, but relies on a very dense exciton gas already giving rise to saturation effects [17]. In such a case, clear frontiers between quantum effects and conventional lasing due to gain arising from an inverted electron-hole population tend to wash out. A similar motivation also applies for a potential condensation of excitons or exciton lasing $[3,18]$. On the device side, a comprehensive study of the MT and thus of the electronic characteristics of III-nitride based quantum wells (QWs) close to the transparency limit should provide essential information about the operating principle and the carrier-injection regime of laser and light-emitting diodes (LEDs). Here, an aspect of particular interest is given by the commonly observed and highly-debated efficiency droop of InGaN-based LEDs occurring at high driving currents $[19,20]$.

In the present Rapid Communication, the combination of a large $E_{\mathrm{X}}^{\mathrm{b}}$ with a state-of-the-art linewidth $\left(\sim 0.25 E_{\mathrm{X}}^{\mathrm{b}}\right)$ in a single $c$-plane GaN QW allows to probe the optically induced MT between 4 and $150 \mathrm{~K}$. The investigated 3.2-nm-thick QW was grown by metal-organic vapor phase epitaxy on a GaN substrate and is sandwiched between two $\mathrm{Al}_{0.09} \mathrm{Ga}_{0.91} \mathrm{~N}$ barriers with top and bottom thicknesses of 150 and $30 \mathrm{~nm}$, respectively. Its fundamental state is composed of two excitonic transitions $\mathrm{X}_{\mathrm{A}}$ and $\mathrm{X}_{\mathrm{B}}$ located around $3.5 \mathrm{eV}$ as evidenced by the photoluminescence excitation (PLE) measurements displayed in Fig. 1(a) [21]. Both excitons feature a similar oscillator strength and a linewidth $\gamma_{\mathrm{X}}=8 \mathrm{meV}$, the latter being equal to their energy separation. At higher energies the bandto-band (BB) absorption edge, the merged longitudinal-optical (LO) phonon replica of the excitons, and eventually a strong PLE intensity increase due to absorption in the barriers can be identified. A direct conclusion on $E_{\mathrm{X}}^{\mathrm{b}}$ from PLE is hampered by the increased broadening of the band edge. However, using the Schrödinger-Poisson (SP) solver described in Ref. [22] we deduce $E_{\mathrm{X}}^{\mathrm{b}}=30 \mathrm{meV}$ based on a normalized zero-density wave-function overlap of 0.57 [cf. Fig. 1(b)]. This value sets the BB position marked in Fig. 1(a), which coincides well with the steepest rise in absorption. The strongly reduced overlap value results from the quantum-confined Stark effect (QCSE) present in polar III-nitride heterostructures due to built-in 

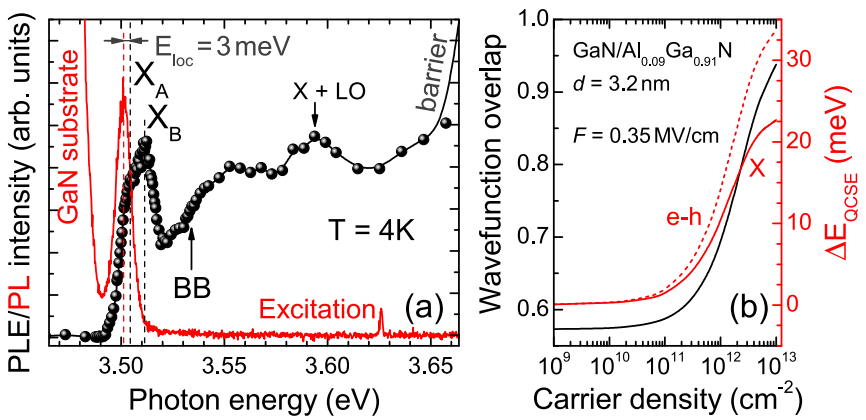

FIG. 1. (Color online) (a) PLE spectrum (black dots) of the QW recorded at $4 \mathrm{~K}$ revealing two fundamental excitons $X_{A}$ and $X_{B}$ and the $\mathrm{BB}$ absorption at higher energies. A PL spectrum (red, excitation energy $3.627 \mathrm{eV}$ ) is shown for comparison. (b) SP simulations: normalized wave-function overlap (black) and QW ground-state energy shift for excitons (red solid, accounting for $E_{\mathrm{X}}^{\mathrm{b}}(n)$ ) and free carriers (red dashed) as a function of $n$.

polarization fields [23]. In addition to the narrow exciton linewidth, the high quality of the present $\mathrm{QW}$ is evidenced by a small localization energy $E_{\mathrm{loc}}=3 \mathrm{meV}$ obtained by comparing the PLE spectrum to the photoluminescence (PL) signal. Following Ref. [24], we deduce $a_{\mathrm{B}}=2.7 \mathrm{~nm}$ for the present QW and thus a hard-sphere limit for $n_{\text {crit }}$ of $4.4 \times 10^{12} \mathrm{~cm}^{-2}$.

The first set of experiments relies on high-power nonresonant time-resolved PL (tr-PL) [21]. Figure 2(a) shows
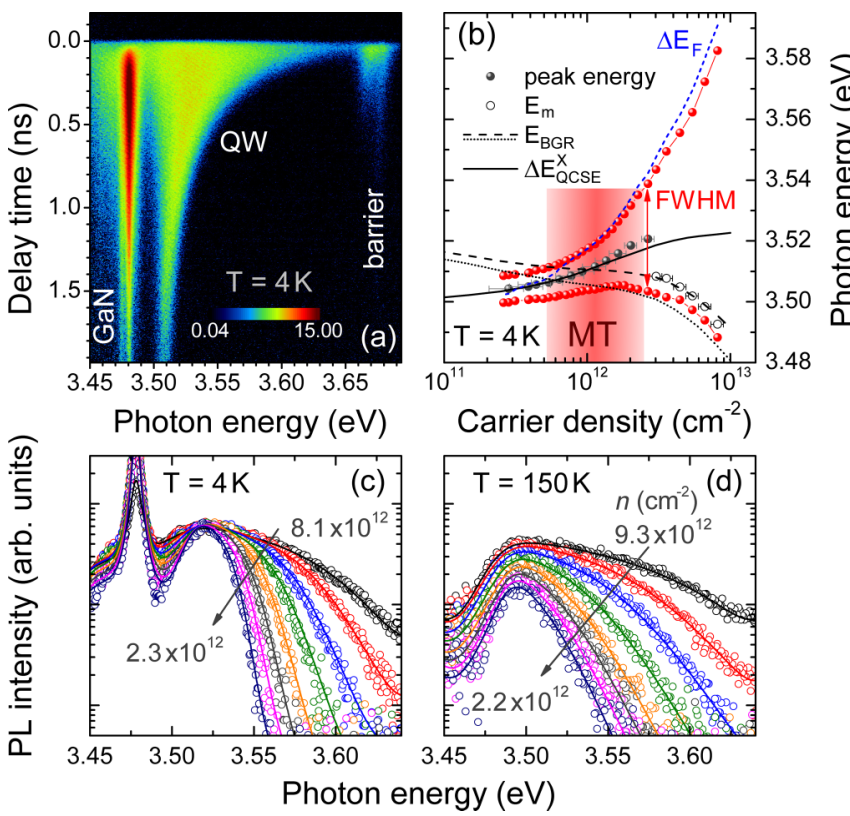

FIG. 2. (Color online) (a) Streak camera image recorded at $4 \mathrm{~K}$. (b) Fingerprint of the MT at $4 \mathrm{~K}$ : evolution of peak energy, upper and lower half-maximum energy (red dots), $E_{\mathrm{m}}, \Delta E_{\mathrm{QCSE}}^{\mathrm{X}}, \Delta E_{\mathrm{F}}$ (blue dashed line), and $E_{\mathrm{BGR}}$ (black dashed line, the result of cw-PL is shown for comparison as a dotted line, cf. Fig. 3) as a function of $n$. The MT range is shaded in red. (c) and (d) Spectral profiles at different time delays: comparison of experimental data (dots) and modeling (lines) at 4 (steps of $60 \mathrm{ps}$ ) and $150 \mathrm{~K}$ (steps of $180 \mathrm{ps}$ ), respectively. a streak camera image recorded at $T=4 \mathrm{~K}$, the excitation pulse arrives at zero delay time with a peak power density of about $20 \mathrm{MW} / \mathrm{cm}^{2}$. The contribution of the QW can be clearly identified between the signal of the GaN substrate around $3.48 \mathrm{eV}$ and the barrier around $3.68 \mathrm{eV}$. Directly after the pulse, the QW luminescence emerges with a large spectral extension $(\sim 100 \mathrm{meV})$, which progressively shrinks down with increasing delay time, while exhibiting a constant peak amplitude. Only when the spectral extension has almost reached its minimum after $\sim 1$ ns delay, the amplitude of the QW emission also starts diminishing. The substrate emission shows a much longer rise time than barrier and QW. Its time scale coincides with the rapid decay of the barrier. Indeed, the total AlGaN layer thickness corresponds to about twice the penetration depth at the pumping wavelength, which makes carrier transfer and photon recycling the main source of recombining carriers in the substrate.

The streak camera images obtained at different $T$ have been analyzed following the model introduced by Kappei et al. in Ref. [11]. The QW emission spectra at various time delays are fitted by convoluting the QW joint density of states (JDOS) weighted by electron and hole quasi-Fermi distributions with a Landsberg-type Lorentzian to account for interparticle scattering $[21,25]$. In the present case, the steplike JDOS has been additionally convoluted with a Gaussian of $8-\mathrm{meV}$ linewidth to account for the inhomogeneous broadening. Adopting effective electron and hole masses of $m_{\mathrm{e}}^{*}=0.2 m_{0}$, $m_{\mathrm{A}}^{*}=1.8 m_{0}$, and $m_{\mathrm{B}}^{*}=0.45 m_{0}$ from $k \cdot p$-simulations [22], where $m_{0}$ denotes the free electron mass, leads thus directly to absolute carrier densities [21]. The results of the fitting procedure accurately match the experimental spectra and are displayed for both 4 and $150 \mathrm{~K}$ in Figs. 2(c) and 2(d), respectively. For the low-temperature analysis, an additional Lorentzian has been added at $3.48 \mathrm{eV}$ to account for the substrate emission, that is already quenched at $150 \mathrm{~K}$. At $4 \mathrm{~K}$, the gradual shrinking of the QW-emission linewidth with increasing time delay is clearly resolved. This process is accompanied by a progressive cooling of the EHP as evidenced by the slope of the high-energy tail. Below the fitted range, the emission band recovers a symmetrical line shape at $n \sim 1.5 \times 10^{12} \mathrm{~cm}^{-2}$. From here, the PL amplitude starts to decay while the emission undergoes a slight redshift. The details at $150 \mathrm{~K}$ remain similar, but are much more blurred owing to increased lattice and carrier temperatures, $T$ and $T_{\text {car }}$, respectively.

The above-mentioned modeling is only valid for $n>n_{\text {crit }}$, i.e., when luminescence originates from an EHP. Hence, in order to obtain an absolute carrier density over the whole explored injection range, $n$ has been extrapolated for $n<n_{\text {crit }}$ based on the QW integrated PL intensity $I_{\mathrm{PL}}$ decay assuming: $I_{\mathrm{PL}} \propto n / \tau_{\text {rad }}$. The QCSE impact on the radiative lifetime $\tau_{\text {rad }}$ based on the wave-function overlap in Fig. 1(b) has iteratively been taken into account. Whereas the determination error in $n$ can be estimated to $10 \%$ above $n_{\text {crit }}$, mainly based on the absolute value of $m_{\mathrm{e}}^{*}$ in $\mathrm{GaN}$, the uncertainty in $n$ increases toward lower injection due to the extrapolation procedure.

Figure 2(b) displays several characteristics of the MT at $4 \mathrm{~K}$ : the evolution of the PL peak energy, its full width at half maximum (FWHM) and the determined band-gap energies $E_{\mathrm{m}}$ above $n_{\text {crit }}$ as a function of $n$. Two different 

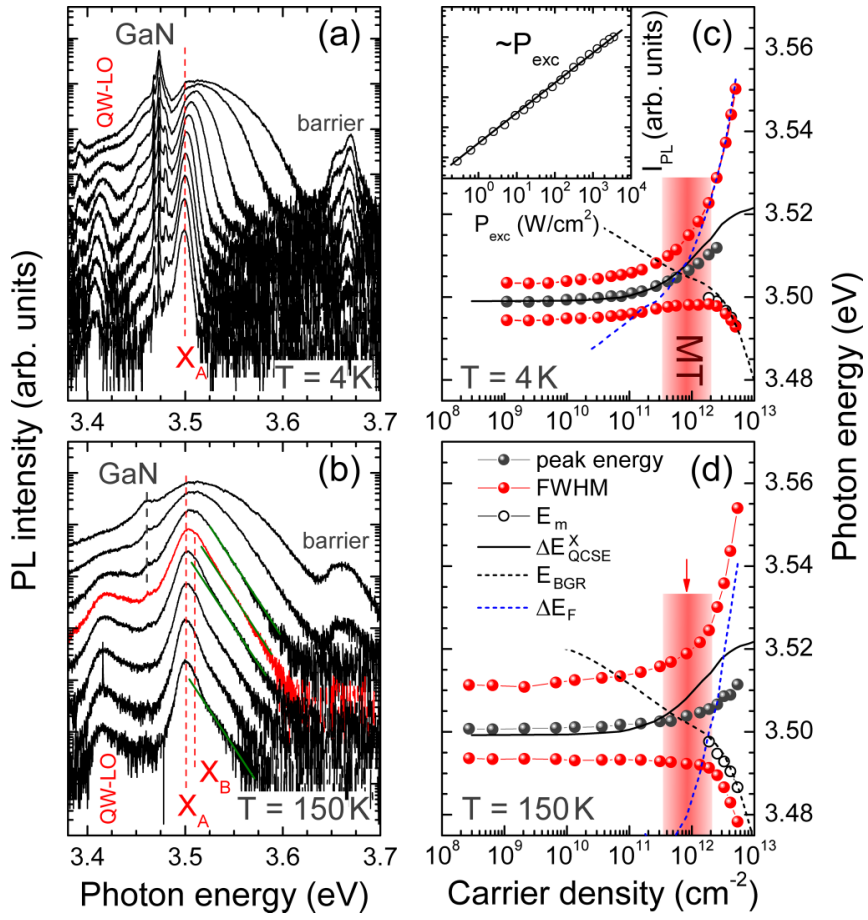

FIG. 3. (Color online) MT in cw-PL: (a) and (b) injectiondependent PL spectra measured at 4 and $150 \mathrm{~K}$, respectively. In (b), exponential fits of the high-energy tail are shown with green lines. The red spectrum marks the transition from a monoexponential tail to an excitonic line shape. (c) and (d) MT at 4 and $150 \mathrm{~K}$ by analogy with Fig. 2(b), respectively. The MT range is highlighted with a red background and the red arrow in (d) marks the position of the red spectrum in (b). The inset in (c) shows $I_{\mathrm{PL}}$ as a function of excitation power density $P_{\text {exc }}$ at $4 \mathrm{~K}$.

injection regimes can be distinguished. For $n<10^{12} \mathrm{~cm}^{-2}$, the emission band is symmetric and blueshifts with rising $n$, while showing a slight increase in the linewidth. This latter effect is ascribed to collisional-induced broadening, which is characteristic for a dense exciton gas [26]. The blueshift occurs due to screening of the internal polarization field, which is estimated to $350 \mathrm{kV} / \mathrm{cm}$ [23]. It can be properly modeled by SP simulations yielding the density-dependent exciton energy $E_{\mathrm{X}}(n)=E_{\mathrm{X}}(0)+\Delta E_{\mathrm{QCSE}}^{\mathrm{X}}$, where $\Delta E_{\mathrm{QCSE}}^{\mathrm{X}}$ comprises the bare free-carrier energy correction $\Delta E_{\mathrm{QCSE}}^{\mathrm{e}-\mathrm{h}}$ and the overlap-dependent rise of $E_{\mathrm{X}}^{\mathrm{b}}$ [cf. free carrier and exciton curves in Fig. 1(b)] [27]. Toward higher carrier densities, the spectral width strongly increases until for $n>3 \times 10^{12} \mathrm{~cm}^{-2}$ the separation of electron and hole quasi-Fermi energies $\Delta E_{\mathrm{F}}$ substantially exceeds the band edge resulting in a broad emission band without any clear peak energy. In this regime, the fitted $E_{\mathrm{m}}$ redshifts with increasing $n$ due to band-gap renormalization (BGR) $[28,29]$. Note that the recorded $E_{\mathrm{m}}$ change is lower than in the forthcoming continuous-wave (cw) PL experiments (cf. results of Fig. 3) and may even be suppressed for the highest $n$ values. This effect appears for all $T$ in tr-PL and is most likely due to feeding of carriers from the $\mathrm{AlGaN}$ barrier-a process disturbing the equilibrium shortly after the pulse. However, for $n>3 \times 10^{12} \mathrm{~cm}^{-2}$ the QW emission is obviously originating from an EHP, while for $n<5 \times 10^{11} \mathrm{~cm}^{-2}$ PL is likely to stem from an exciton gas as no significant change in the emission line shape is observed down to the lowest densities (cf. also Fig. 3). Thus, from the criterion of the bare emission line shape, the range between these two densities corresponds to the crossover between EHP and exciton populations and $n_{\text {crit }}$ at $T=4 \mathrm{~K}$ can be estimated to $(1.5 \pm 1) \times 10^{12} \mathrm{~cm}^{-2}$, i.e., significantly below the hard-sphere limit.

The results of tr-PL are confirmed by the cw-PL measurements displayed in Fig. 3. Using a frequency-doubled $\mathrm{Ar}^{+}$laser $(\lambda=244 \mathrm{~nm})$, the excitation power density could be varied over four orders of magnitude, from 0.3 to $3600 \mathrm{~W} / \mathrm{cm}^{2}$ [21]. The high-injection spectra shown in Fig. 3(a) closely resemble the spectral profiles from Fig. 2(c) and allow applying the same analysis as detailed before for the tr-PL measurements. The results are displayed in Fig. 3(c) and complement the tr-PL data down to lower densities. A significant difference is only marked by the BGR-induced redshift at high densities, which appears much more pronounced than in tr-PL owing to the absence of a relaxation nonequilibrium shortly after the pulse. The band gap can be fitted by the power law $E_{\mathrm{BGR}}=$ $E_{\mathrm{X}}(0)+E_{\mathrm{X}}^{\mathrm{b}}+\Delta E_{\mathrm{OCSE}}^{\mathrm{e}-\mathrm{h}}-\alpha n^{1 / 3}[29,30]$, yielding $\alpha=3.8 \times$ $10^{-6} \mathrm{eVcm}^{2 / 3}$ at $4 \mathrm{~K}-$ a value very close to the prediction of Ref. [30]. The clear observation of BGR allows to identify additional criteria defining the MT: (i) the transparency limit $\Delta E_{\mathrm{F}}=E_{\mathrm{BGR}}$, when the quasi-Fermi level separation exceeds the band edge marking the onset of gain from an EHP [31]. This is a purely fermionic effect which excludes excitonic absorption at the band edge. (ii) The crossing $E_{\mathrm{X}}=E_{\mathrm{BGR}}$, where the exciton merges with the continuum states and $E_{\mathrm{X}}^{\mathrm{b}}$ vanishes $[12,32]$. Both criteria are depicted by the scheme of Fig. 4(a) and yield a value around $7 \times 10^{11} \mathrm{~cm}^{-2}$, coinciding well with the previous line shape criterion indicated by the red background in Fig. 3(c). Note that tr-PL and cw-PL do not necessarily have to yield identical $n_{\text {crit }}$ values: whereas in tr-PL after a certain time delay, exciton screening can only be due to cold carriers, cw excitation results in a static hot carrier population potentially altering the screening efficiency [2].

Apart from the naturally increased linewidth, the characteristics of the MT at higher $T$ [cf. Fig. 3(b) at $150 \mathrm{~K}$ ]
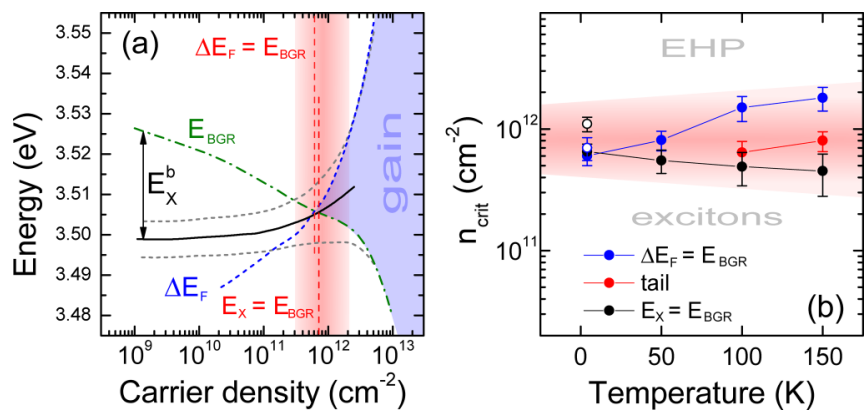

FIG. 4. (Color online) (a) Schematic of the MT-defining criteria $E_{\mathrm{X}}=E_{\mathrm{BGR}}$ and $\Delta E_{\mathrm{F}}=E_{\mathrm{BGR}}$ (based on cw-PL values taken at $4 \mathrm{~K}$ ). The MT range is shaded in red, $E_{\mathrm{X}}$ is given by the black solid line, and the gray dashed lines mark upper and lower QW half maximum energies. (b) $n_{\text {crit }}$ values deduced from various criteria: $\Delta E_{\mathrm{F}}=E_{\mathrm{BGR}}$ (blue), disappearance of an excitonic line shape [red, cf. Fig. 3(d)], and $E_{\mathrm{X}}=E_{\mathrm{BGR}}$ (black). Results of tr-PL at $4 \mathrm{~K}$ are shown with open circles. The red background marks the confidence interval. 
appear slightly modified: one can still note the broad QW emission band at high $n$ with an exponential high-energy tail. But, owing to the increased $T_{\text {car }}$, the emission plateau is now much less pronounced in agreement with the tr-PL results shown in Fig. 2(d). Moreover, a significant occupation of $X_{B}$ and even free carrier states is observed at lower injection as manifested by the high-energy shoulder in the exciton emission profile and the still present exponential high-energy tail far below $n_{\text {crit }}$. Although the band edge cannot be clearly identified [33], the vanishing of the exciton line shape and the transition to a monoexponential high-energy tail marked by the red spectrum in Fig. 3(b) thus set another Mott criterion. Slight changes also emerge for BGR: an increased $\alpha$ value of $4.1 \times 10^{-6} \mathrm{eVcm}^{2 / 3}$ is deduced at $150 \mathrm{~K}$, leading in combination with $T_{\text {car }}$ to the variation of the MT defining criteria displayed in Fig. 4(b), whereas no significant change in $n_{\text {crit }}$ could be traced from pure spectral arguments, the rise of $T_{\text {car }}$ causes the expected increase in the onset of gain $\Delta E_{\mathrm{F}}=E_{\mathrm{BGR}}$ with $T$, which is contrasting with the condition $E_{\mathrm{X}}=E_{\mathrm{BGR}}$, where an increasing $\alpha$ causes a decrease in $n_{\text {crit }}$. Eventually, no clear trend on $n_{\text {crit }}$ can be inferred within the explored $T$ range. At all studied temperatures the MT takes place between 0.5 and $2 \times 10^{12} \mathrm{~cm}^{-2}$, i.e., more than one order of magnitude above the $n_{\text {crit }}$ value of InGaAs-based QWs [11].

At first glance, the absence of a distinct MT temperature dependence seems to contradict the simple interpretation of an increasing static screening length as employed in Ref. [8]. However, it is worth recalling that the screening efficiency may be influenced by dynamical effects [15,34], also depending on the ratio $k_{\mathrm{B}} T / E_{\mathrm{X}}^{\mathrm{b}}$ [2], which remains significantly below unity in this study contrary to that of Ref. [8]. In the end, despite the different dimensionality our results agree well with the findings and considerations of Klingshirn et al. for $\mathrm{ZnO}$ [3], where $n_{\text {crit }}$ is defined by BGR and thus features only a weak $T$ dependence.
Before concluding, another important aspect of the present study shall briefly be highlighted. The strict linearity of $I_{\mathrm{PL}}$ with increasing pump power shown in the inset of Fig. 3(c) and the absence of any ultra-fast initial decay in tr-experiments [21] exclude Auger-scattering as a nonradiative loss process in the present QW up to densities as high as $5 \times 10^{12} \mathrm{~cm}^{-2}$, i.e., far beyond the Bernard-Duraffourg limit [31]. It is worth mentioning that this statement does not necessarily need to apply directly for InGaN-based LEDs, even though they are supposed to operate at much lower carrier densities. Indeed, Augereffects occurring in In-containing QWs might be significantly enhanced, e.g., due to the lower band gap and the strongly increased carrier lifetime [19,20]. Nevertheless, we suggest that equivalent studies performed on appropriate structures could be used as a powerful tool to further assess the potential role played by Auger-recombination in InGaN-based LEDs. They allow a precise determination of the QW carrier concentration via monitoring the injection-dependent emission line shape, that can be readily compared with theoretical models.

In conclusion, the Mott transition in a polar GaN-based QW has been studied over a significant range of lattice temperatures using nonresonant optical carrier injection. It was invariably found to be connected to a strong modification in the QW emission spectrum and to occur at constant QW carrier densities around $10^{12} \mathrm{~cm}^{-2}$ whatever the temperature, which contradicts a simplistic interpretation in terms of static screening. The absence of any obvious signature of the Augerprocess up to the transparency regime in the present GaN QW should bring new insights into the issue of the efficiency droop occurring at high current densities in InGaN-based QW LEDs, which still remains debated.

This work was supported by the NCCR Quantum Photonics, research instrument of the Swiss National Science Foundation (SNSF), and by the SNSF (Grant Nos. 200020-113542 and 200020-153620).
[1] N. F. Mott, Philos. Mag. 6, 287 (1961).

[2] S. Schmitt-Rink, D. S. Chemla, and D. A. B. Miller, Phys. Rev. B 32, 6601 (1985).

[3] C. Klingshirn, R. Hauschild, J. Fallert, and H. Kalt, Phys. Rev. B 75, 115203 (2007).

[4] N. F. Mott, Rev. Mod. Phys. 40, 677 (1968).

[5] L. M. Sander and D. K. Fairobent, Solid State Commun. 20, 631 (1976).

[6] L. J. Schowalter, F. M. Steranka, M. B. Salamon, and J. P. Wolfe, Phys. Rev. B 29, 2970 (1984).

[7] J. Shah, M. Combescot, and A. H. Dayem, Phys. Rev. Lett. 38, 1497 (1977).

[8] H. Schweizer, A. Forchel, A. Hangleiter, S. Schmitt-Rink, J. P. Löwenau, and H. Haug, Phys. Rev. Lett. 51, 698 (1983).

[9] F. Binet, J. Y. Duboz, J. Off, and F. Scholz, Phys. Rev. B 60, 4715 (1999).

[10] H. W. Yoon, M. D. Sturge, and L. N. Pfeiffer, Solid State Commun. 104, 287 (1997).

[11] L. Kappei, J. Szczytko, F. Morier-Genoud, and B. Deveaud, Phys. Rev. Lett. 94, 147403 (2005).
[12] G. Manzke, D. Semkat, and H. Stolz, New J. Phys. 14, 095002 (2012).

[13] Note that there are also experimental results that indicate an abrupt MT in QWs, e.g., in Ref. [16]. However, the latter study deals with coupled QWs and indirect excitons, which increases the complexity of the problem and hampers a direct comparison. This statement is illustrated by the fact that in Ref. [16] the MT of indirect excitons could exclusively be observed in presence of direct excitons acting as catalystlike species.

[14] With $E_{\mathrm{X}}^{\mathrm{b}} \approx 2 \mathrm{meV}$ in bulk Ge, the limited stability of excitons is severely restricting the accessible $T$ range.

[15] S. Ben-Tabou de-Leon and B. Laikhtman, Phys. Rev. B 67, 235315 (2003).

[16] M. Stern, V. Garmider, V. Umansky, and I. Bar-Joseph, Phys. Rev. Lett. 100, 256402 (2008).

[17] J. Kasprzak, M. Richard, S. Kundermann, A. Baas, P. Jeambrun, J. M. J. Keeling, F. M. Marchetti, M. H. Szymanska, R. André, J. L. Staehli, V. Savona, P. B. Littlewood, B. Deveaud-Plédran, and L. S. Dang, Nature (London) 443, 409 (2006). 
[18] J. Ding, H. Jeon, T. Ishihara, M. Hagerott, A. V. Nurmikko, H. Luo, N. Samarth, and J. Furdyna, Phys. Rev. Lett. 69, 1707 (1992).

[19] M. H. Kim, M. F. Schubert, Q. Dai, J. K. Kim, E. F. Schubert, J. Piprek, and Y. Park, Appl. Phys. Lett. 91, 183507 (2007).

[20] E. Kioupakis, P. Rinke, K. T. Delaney, and C. G. Van de Walle, Appl. Phys. Lett. 98, 161107 (2011).

[21] See Supplemental Material at http://link.aps.org/supplemental/ 10.1103/PhysRevB.90.201308 for a description of (i) the experimental details, (ii) the EHP emission modeling, and (iii) the carrier dynamics.

[22] G. Rossbach, J. Levrat, E. Feltin, J.-F. Carlin, R. Butté, and N. Grandjean, Phys. Rev. B 88, 165312 (2013).

[23] M. Leroux, N. Grandjean, M. Laugt, J. Massies, B. Gil, P. Lefebvre, and P. Bigenwald, Phys. Rev. B 58, R13371 (1998).

[24] X. F. He, Phys. Rev. B 43, 2063 (1991).

[25] P. T. Landsberg, Phys. Status Solidi 15, 623 (1966).
[26] B. Deveaud, F. Clérot, N. Roy, K. Satzke, B. Sermage, and D. S. Katzer, Phys. Rev. Lett. 67, 2355 (1991).

[27] P. Bigenwald, A. Kavokin, B. Gil, and P. Lefebvre, Phys. Rev. B 63, 035315 (2001).

[28] L. Hedin, Phys. Rev. 139, A796 (1965).

[29] D. A. Kleinman and R. C. Miller, Phys. Rev. B 32, 2266 (1985).

[30] S. Schmitt-Rink, C. Ell, S. W. Koch, H. E. Schmidt, and H. Haug, Solid State Commun. 52, 123 (1984).

[31] M. G. A. Bernard and G. Duraffourg, Phys. Status Solidi 1, 699 (1961).

[32] R. Zimmermann, Phys. Status Solidi B 146, 371 (1988).

[33] Similarly to Ref. [11], we could not identify any clear evidence of BGR below the critical density. This hints at a more abrupt loss of $E_{\mathrm{X}}^{\mathrm{b}}$ across the MT than would be expected from the BGR density scaling.

[34] S. Schmitt-Rink and C. Ell, J. Lumin. 30, 585 (1985). 